\title{
An auto-focusing approach for a dynamic quadrature interferometer
}

Gollwitzer, Andreas; Haugg, Susanne; Fischerauer, Gerhard

Lehrstuhl für Mess- und Regeltechnik, Universität Bayreuth

D-95540 Bayreuth

\begin{abstract}
The measurement of surface vibrations with small amplitudes is often accomplished using interferometric techniques. For a good local resolution of the vibration amplitudes, the laser beam has to be focused on the surface through a high magnification lens. Owing to the small depth of focus of this lens, its distance to the surface has to be controlled accurately and precisely. In order to avoid the nonlinear components present in heterodyne interferometers (such as acousto-optic modulators) and to simultaneously eliminate the effects of noise sources such as fluctuations in the surface reflectivity of the device under test, we use a homodyne setup with additional path length modulation. The sensitivity of the setup is increased by quadrature interferometry, i.e., the synchronous measurement of the intensities detected by two photodiodes. We also present a signal processing technique for the control of the distance between lens and surface; this makes sure that the laser beam is optimally focused onto the surface and leads to a high overall signal-to-noise ratio.
\end{abstract}

\section{Introduction}

The detection of vibrations with amplitudes in the subnanometer range as they appear in connection with surface acoustic waves (SAW) in solids is a challenging task which can be solved by optical methods [1, 2]. We have shown previously that a homodyne Michelson interferometer is capable of observing SAWs at frequencies of about $100 \mathrm{MHz}$ and amplitudes down to $30 \mathrm{pm}$ [3]. With a special acquisition system and additional signal processing, this homodyne interferometer is able to extract the absolute amplitude and phase of the vibrations [4]. Owing to the path length modulation realized by a piezoactuator placed on the backside of the reference mirror, the system reaches its optimal working point only during part of the measurement time. The algorithm for extraction of the vibration phase described in [5] uses all measurement values, but the same is not true for the amplitude evaluation algorithm. The present work aims at an optimization of the latter algorithm.

Another problem unsolved in this context is the automatic focusing of the laser beam onto the device under test (DUT) surface. We investigated a novel solution to this problem based on quadrature interferometry and resulting in a much higher overall signal-to-noise ratio.

\section{Optical Setup}

The setup of our quadrature interferometer is shown in Fig. $1[6,7]$. The beam of a linearly polarized laser (Melles-Griot 25 LHP 925, wavelength $\lambda=632.8 \mathrm{~nm}$, output power $P_{0}=17 \mathrm{~mW}$ ) is split into a measuring and a reference beam by a polarizing beam splitter (PBS). By two $\lambda / 4$ retardation plates with the fast axis at $45^{\circ}$, the plane of polarization of the reflected beam is rotated out of plane and thus prevented from reentering into the laser [8]. As the reference and the measurement beams are polarized perpendicularly to each other, one of them is easily delayed with a third $\lambda / 4$ retardation plate. With a linear analyzer rotated by $45^{\circ}$, an interference effect is created, which can be detected by the photodiodes (Newport 818BB-21A). When the DUT surface vibrates sinusoidally, the truncated Fourier series of the intensity measured by the two photodiodes is given by [9]

$$
\begin{aligned}
& I_{\mathrm{PD} 1} \approx I_{01}+\hat{I}_{1} \cdot\left(\cos \tilde{z} \cdot J_{0}(\tilde{a})-2 \sin \tilde{z} \cdot J_{1}(\tilde{a}) \cdot \cos \left(\omega t+\varphi_{0}\right)-\ldots\right), \\
& I_{\mathrm{PD} 2} \approx I_{02}-\hat{I}_{2} \cdot\left(\sin \tilde{z} \cdot J_{0}(\tilde{a})+2 \cos (\tilde{z}) \cdot J_{1}(\tilde{a}) \cdot \cos \left(\omega t+\varphi_{0}\right)-\ldots\right) .
\end{aligned}
$$

Here, $\tilde{z}=z \cdot 4 \pi / \lambda$ and $\tilde{a}=a \cdot 4 \pi / \lambda$ are the normalized reference mirror and DUT displacements, respectively. $l_{0 i}$ and $\hat{l}_{i}$ denote the bias intensities and intensity amplitudes, respectively, at the two photodiodes, and $J_{v}(z)$ is the $v$-order Bessel function of the first kind. 


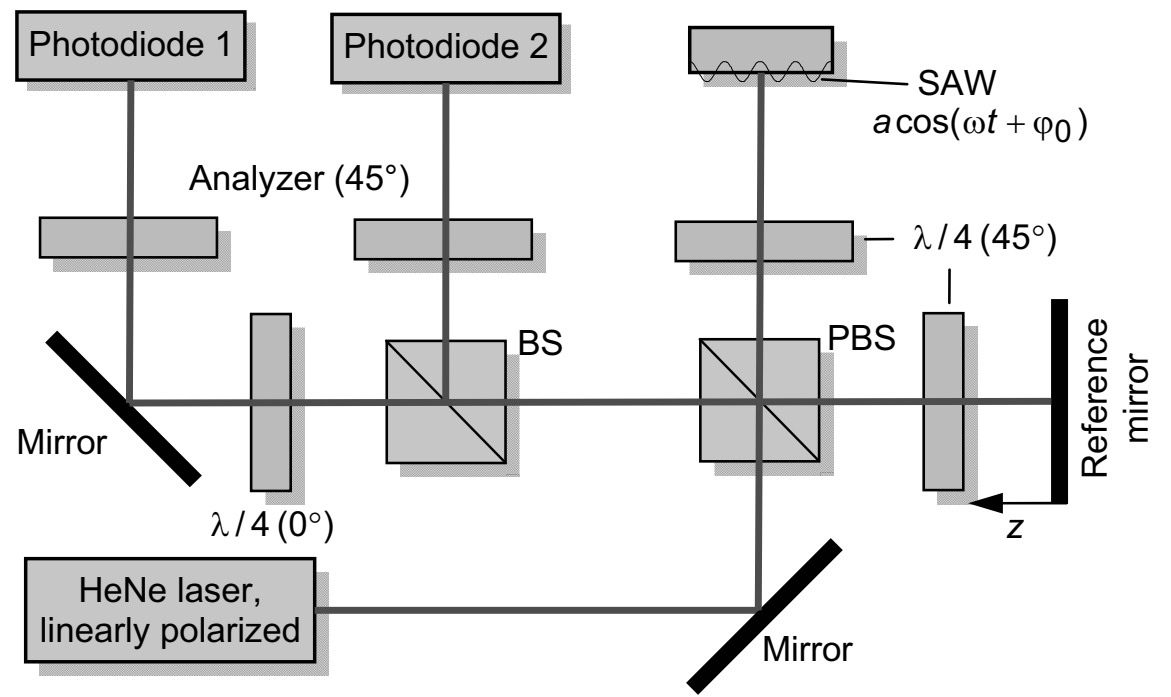

Figure 1. Optical setup of the quadrature interferometer.

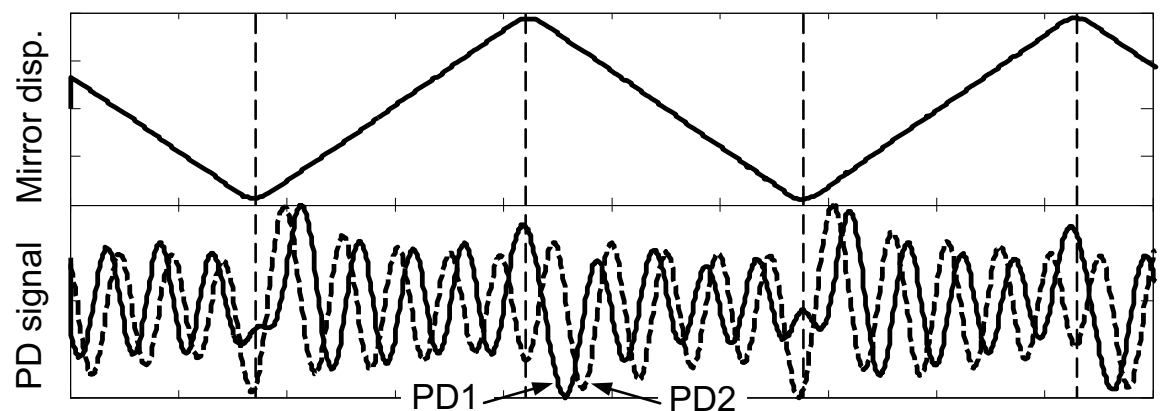

Figure 2. Reference mirror displacement and corresponding measurement signals of the two photodiodes.

The measured output signals of the two photodiodes are shown in Fig. 2 for the case of a triangular reference mirror vibration at an amplitude of a few micrometers. These data agree with equation (1). The photodiode signals effectively correspond to the in-phase and the quadrature components of the interference signal as a function of the reference mirror displacement. The phase shift between the two periodic signals can be used to determine the direction of the reference mirror movement. It is notable that the sensitivity of the interferometer to the high-frequency vibration on the DUT, which can be expressed by the slope of the photodiode signals according to equation (1), does not vanish simultaneously in both signals. Hence, every measured point contributes towards the information on the DUT vibration amplitude and phase.

\section{Electrical Setup}

The excitation frequencies of SAW devices and the reference mirror differ by about seven orders $(\sim 100 \mathrm{MHz}$ vs. $10 \mathrm{~Hz})$. Therefore, the respective contributions to the intensity spectrum lie in completely different frequency ranges. This fact is taken into account by employing two different acquisition systems. The low-frequency signal due to the mirror displacement is monitored by a high-bandwidth digital sampling oscilloscope (DSO, Agilent 54832B) which captures every nonlinearity created by the interferometer. The high-frequency signal due to the DUT is measured by a vector network analyzer (VNA, Agilent N5230A). The latter is also used to excite SAWs via the usual interdigital transducers (IDT), comblike electrodes placed on piezoelectric substrates and acting as electro-acoustic transducers [10]. It has a very small bandwidth and can measure signals with very small amplitudes like a lock-in amplifier. In addition, it records the amplitudes and phases of the photodiode signals relative to the excitation signal.

The logical structure of the system is depicted in Fig. 3. The acoustic waves excited by the VNA modulate the light intensity in the interferometer. These light intensity fluctuations are converted back to electrical signals by the photodiodes and returned to the VNA. At the same time, the DSO tracks the displacement of the mirror in the reference arm of the interferometer. This serves to eliminate the influence of DUT reflectivity and to determine the absolute acoustic wave amplitude [4]. 


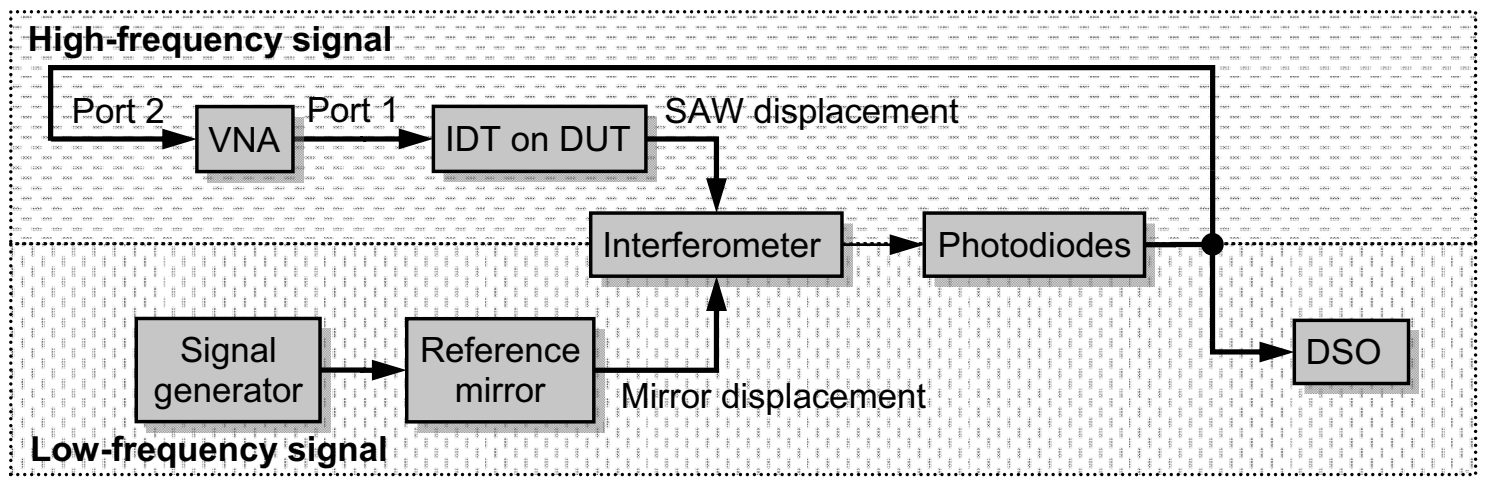

Figure 3. Functional diagram of the measurement system. The diagram does not show the motor stages by which the DUT can be moved such that a surface scan becomes possible.

\section{Signal Processing}

To improve the signal-to-noise ratio, we usually process 20 subsequent measurements by the VNA and 40 subsequent measurements by the DSO for each photodiode. Because the photodiode signals are in quadrature, they will produce an ellipsis when plotted as an xy-plot. This is shown in Fig. 4a for the lowfrequency part of the signals (measured by the DSO). The 40 scattered measurement points are approximated by an ellipse with semiaxes

$$
u_{\mathrm{DSO}} \approx \hat{l}_{1} J_{0}(\tilde{a}) \approx \hat{l}_{1} \text { and } v_{\mathrm{DSO}} \approx \hat{l}_{2} J_{0}(\tilde{a}) \approx \hat{l}_{2} .
$$

In an analogous fashion, the 20 measurement points obtained from the VNA are approximated by an ellipse with semiaxes

$$
u_{\mathrm{VNA}} \approx 2 \hat{l}_{1} J_{1}(\tilde{a}) \approx \hat{l}_{1} \tilde{a} \text { and } v_{\mathrm{VNA}} \approx \hat{l}_{2} 2 J_{1}(\tilde{a}) \approx \hat{l}_{2} \tilde{a}
$$

Here, the small-signal approximation to the Bessel function holds because the SAW amplitude is very small compared to the laser wavelength [11].

The wanted vibration amplitude can be extracted from either one of the above equation sets:

$$
a=\frac{\lambda}{4 \pi} \frac{u_{\mathrm{VNA}}}{u_{\mathrm{DSO}}}=\frac{\lambda}{4 \pi} \frac{v_{\mathrm{VNA}}}{v_{\mathrm{DSO}}}
$$

This redundancy is used to check the accuracy of the setup.

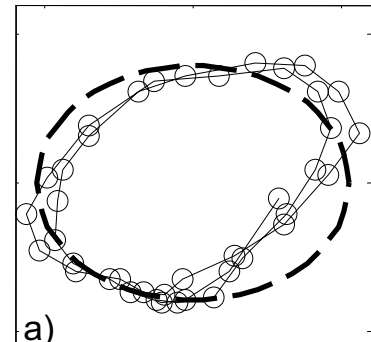

a)

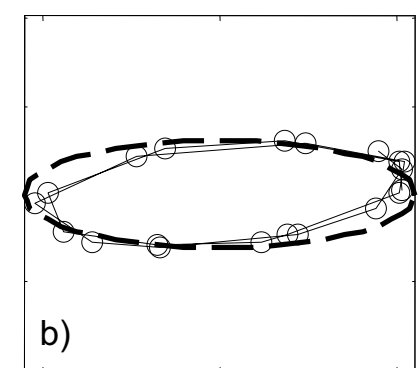

Figure 4. Phasor endpoints obtained from a) DSO and b) VNA output (circles) and approximating ellipses (dashed).

\section{Auto-Focusing Technique}

A problem of major practical importance is the optimal focusing of the laser spot onto the surface of the DUT. For a small spot size, one has to use a lens with a small focal length $f$ (we used a microscope objective Leitz-Wetzlar PLAN 125x/0.8). This implies that the depth of focus ist very short, and the distance between DUT surface and lens has to be tightly controlled. Fig. $5 b$ shows an example for the effects of a bad focusing: the dark grey at the top and bottom of the scanned area indicate a signal-tonoise ratio so low that equation (3) cannot be evaluated any longer 


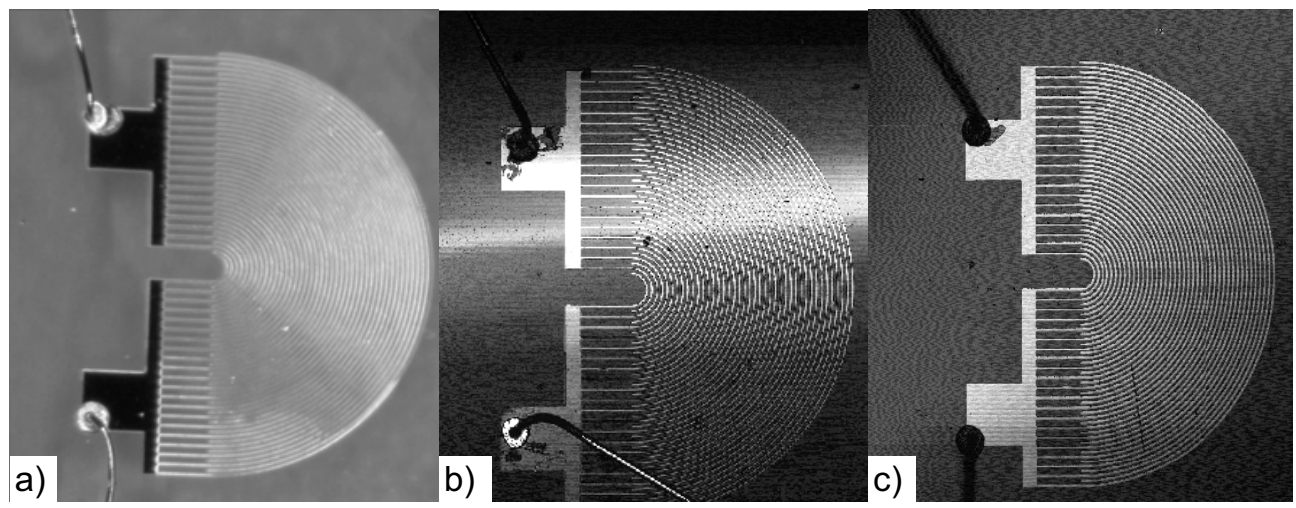

Figure 5. Influence of the focusing on the signal $u_{\mathrm{DSO}}$. a) Microscope view of the DUT surface. b) Measurement result with bad focusing. c) Measurement result with good focusing.
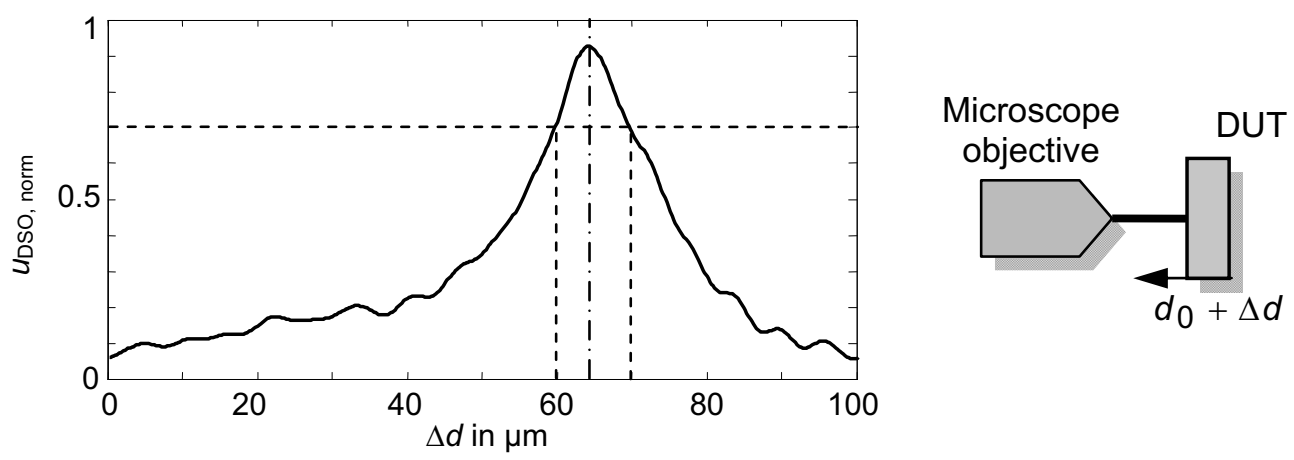

Figure 6. Normalized DSO output $u_{\mathrm{DSO}}$ as a function of distance changes $\Delta d$ between lens and DUT surface.

A plot of the DSO output signal $u_{\text {DSO }}$ as a function of the distance between lens and DUT surface reveals that the deviation from the optimum distance must not exceed a few micrometers for the defocusing to be negligible (Fig. 6). This calls for an automatic focusing technique, which, however, cannot be based on the signal $u_{\mathrm{Dso}}$ alone as it does not contain information about the direction into which one has to move the DUT to bring it back into focus (one value of $u_{\text {Dso }}$ corresponds to two values of the distance $\Delta d$ ).

We implemented an auto-focusing technique based on the observation that the two photodiodes in the quadrature interferometer deliver their maximum output signal at different lens-to-DUT distances $d$ (Figs. 7 and 8). The geometrical path length difference for the light detected by the photodiodes is $\Delta b=14 \mathrm{~cm}$. This corresponds to a lens-to-DUT distance difference of $\Delta d=3 \mu \mathrm{m}$. Hence, the ratio $r$ of the two photodiode output signals contains unambigous information about the direction of the required lens movement. In this manner, the distance can be controlled in a range of about $10 \mu \mathrm{m}$ (Fig. 8b).
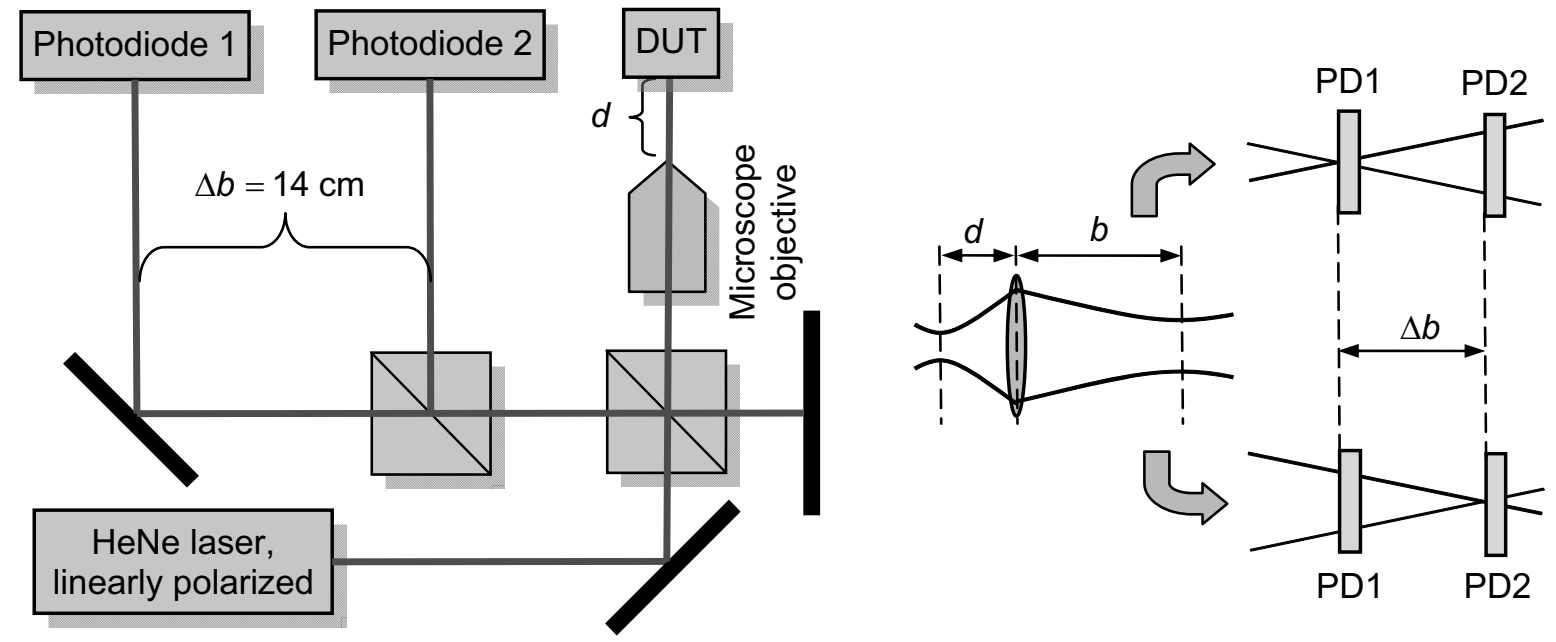

Figure 7. Principle of auto-focusing technique. 


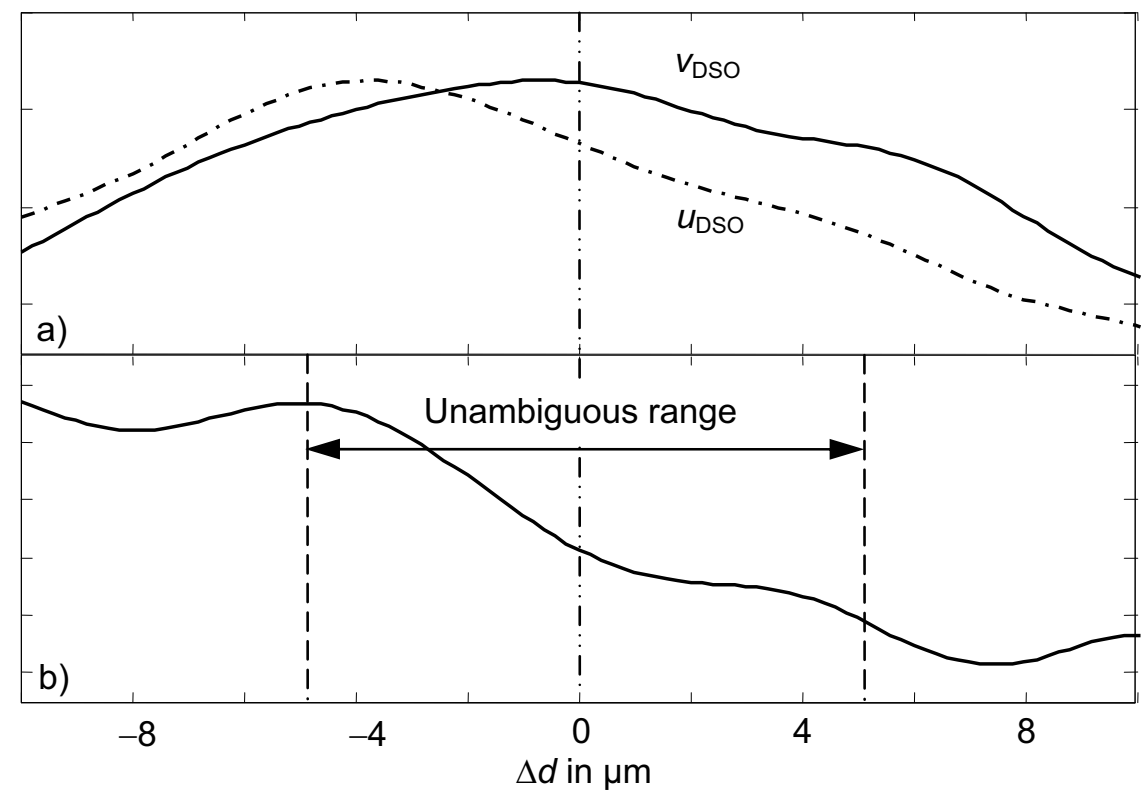

Figure 8. a) In-phase and quadrature DSO signals $u_{\text {Dso }}$ and $v_{\text {DSO }}$ (smoothed and normalized) as a function of the change $\Delta d$ in the lens-to-DUT distance. b) Computed focus control signal $r=u_{\mathrm{DSO}} / v_{\mathrm{DSO}}$.

\section{Experimental Results}

The measurement system was validated by the observation of surface acoustic waves excited by the semicircular IDT shown in Fig. 5a. The aluminum thin-film IDT was patterned onto a piezoelectric substrate $\left(Y, Z\right.$-LiNbO $\left.{ }_{3}\right)$ and comprised 41 fingers with a width of $8 \mu \mathrm{m}$ each. The magnitude of the VNA output signal $U_{\mathrm{VNA}}$ as recorded at 243000 points in an area of size $1.8 \mathrm{~mm} \times 2.16 \mathrm{~mm}$ in the vicinity of the IDT is shown in Fig. 9. As this signal depends on the vibration amplitude a as well as on the surface reflectivity, the structure of the metallic IDT becomes visible in this plot. These measurement results agree well with simulation results based on the so-called delta-function model $[4,12]$.

To study the applicability of equation (3), other measurements were carried out with the IDT patterned onto a $128^{\circ} \mathrm{rotY}, \mathrm{X}-\mathrm{LiNbO}_{3}$ substrate. The DSO signal $u_{\mathrm{Dso}}$ does not depend on a and only mirrors the surface reflectivity difference between the piezoelectric substrate and the metallic IDT (Fig. 10a). In contrast, the VNA signal $u_{\mathrm{VNA}}$ depends on both a and the surface reflectivity (Fig. 10b). And, by equation (3), the ratio of the two signals yields the vibration amplitude a (Fig. 10c). The results clearly demonstrate that the influence of the reflectivity could be suppressed and that the undisturbed absolute vibration amplitude could be extracted. In the present case, amplitudes of up to $500 \mathrm{pm}$ were detected.

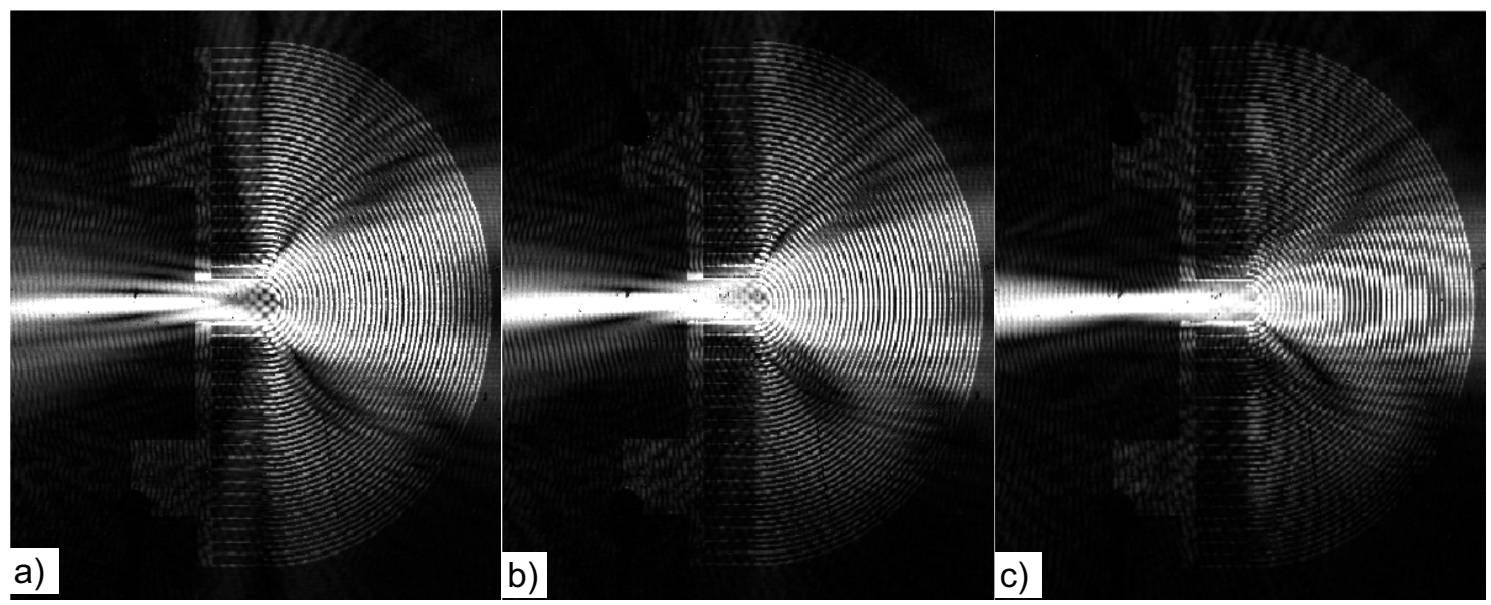

Figure 9. Experimentally observed surface acoustic waves launched multidirectionally by a semicircular IDT on a $Y, X$ - $\mathrm{LiNbO}_{3}$ substrate (pseudocolor plot of vibration amplitude). Excitation frequency: a) $117 \mathrm{MHz}$, b) $118 \mathrm{MHz}$, and c) $120 \mathrm{MHz}$. 


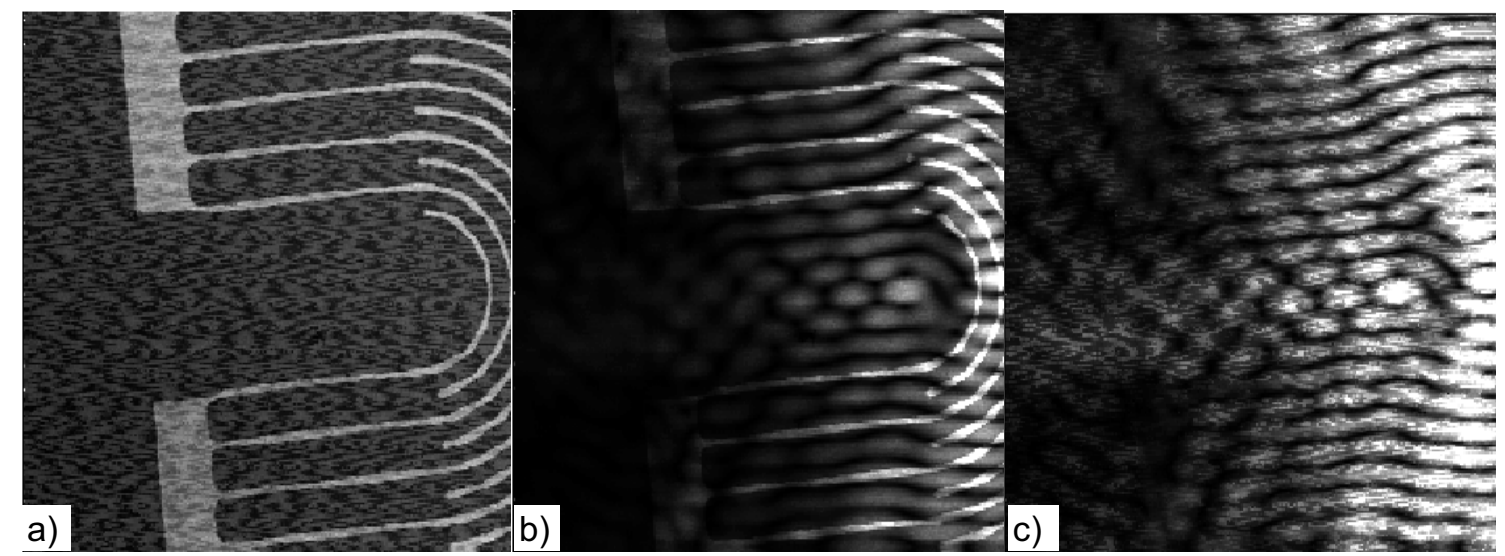

Figure 10. Experimentally observed surface acoustic waves on $128^{\circ} \operatorname{rot} Y, X$ - $\mathrm{LiNbO}_{3}$ (IDT with same layout as in Fig. 9, excited at $120 \mathrm{MHz}$ ). a) DSO signal $u_{\mathrm{DSO}}$. b) VNA signal $u_{\mathrm{VNA}}$. c) SAW amplitude $a$.

\section{Discussion and Conclusion}

We have demonstrated a method based on quadrature interferometry by which absolute vibration amplitudes in the high $\mathrm{MHz}$ or even $\mathrm{GHz}$ range can be determined. An appropriate signal processing algorithm suppresses the effects of reflectivity changes on the observed surface. In addition, the automatic focus control implemented in the system makes sure that long-term measurements such as highresolution surface scans can be carried out without the risk of intermittent defocusing problems. The method has been validated with surface acoustic wave devices on $\mathrm{LiNbO}_{3}$. The measurements clearly render standing and traveling microacoustic waves and agree well with simulations.

It remains to be shown that the reflectivity-insensitive, auto-focusing quadrature interferometer is capable of measuring not only the absolute amplitude, but also the absolute phase of high-frequency surface vibrations. One possible approach is to transfer the phase extraction method from the homodyne interferometer in [4] to the present setup.

\section{$\underline{\text { References }}$}

[1] H. E. Engan and A. Ronnekleiv, "Enhancement of SAW laser probe measurements by signal processing," IEEE Ultrasonics Symposium, Caesars Tahoe, Nevada USA, pp. 217-220, 1999.

[2] K. Kokkonen, J.V. Knuuttila, V.P. Plessky, and M.M. Salomaa, "Phase-sensitive absolute-amplitude measurement of surface waves using heterodyne interferometry," Proc. IEEE Ultrasonics Symposium, Honolulu, Hawaii USA, pp. 1145-1148, 2003.

[3] A. Gollwitzer and G. Fischerauer, "A Novel Method for the Visualization of Vibrations in Surface Acoustic Wave Devices," Proc. Sensor Conference 2007, Vol. II, Nuremberg, pp. 81-86, 2007.

[4] A. Gollwitzer and G. Fischerauer, "Absolute amplitude and phase measurement for microacoustic vibrations based on calibration-free homodyne interferometry," Proc. Eurosensors XXII, Dresden, Germany, pp. 648-651, 2008

[5] A. Gollwitzer and G. Fischerauer, "Interferometric Observation of Surface Acoustic Wave Phase Fronts," Proc. IEEE International Frequency Control Symposium, Miami, USA, pp. 424-427, 2006.

[6] C.B. Scruby and L.E. Drain, Laser ultrasonics. London: Hilger, 1990.

[7] Ju-Yi Lee and Sing-Sian Cai, "A phase quadrature interferometer for measuring the small optical rotation angle of a chiral medium," Optics Communications 281, pp. 2735-2739, 2008

[8] C. Chao, Z. Wang, W. Zhu, and O. Tan, "Scanning homodyne interferometer for characterization of films and microelectromechanical systems devices," Review of scientific instruments, Vol. 76, No. 6, 063906, 2005.

[9] A. Gollwitzer and G. Fischerauer, "Calibration-free non-contact measurement of nano-amplitudes of $\mathrm{MHz}$ vibrations," Proc. ICICI Bandung, Indonesia, 2005

[10] R.M. White, F.W. Voltmer, "Direct piezoelectric coupling to surface elastic waves," Appl. Phys. Lett., Vol. 7, pp. 314-316, 1965.

[11]M. Abramowitz and I. A. Stegun, Handbook of Mathematical Functions. Washington: US Department of Commerce, pp. 369-370 1968.

[12]D. P. Morgan, Surface-Wave Devices for Signal Processing. Amsterdam: Elsevier, pp. 57-88, 1991. 